\title{
$\iota$ Horologi, the first coronal activity cycle in a young solar-like star ${ }^{\star}$
}

\author{
J. Sanz-Forcada ${ }^{1}$, B. Stelzer ${ }^{2}$, and T. S. Metcalfe ${ }^{3,4}$ \\ ${ }^{1}$ Departamento de Astrofísica, Centro de Astrobiología (CSIC-INTA), ESAC Campus, PO Box 78, 28691 Villanueva de la Cañada, \\ Madrid, Spain \\ e-mail: jsanz@cab.inta-csic.es \\ 2 INAF - Osservatorio Astronomico di Palermo G. S. Vaiana, Piazza del Parlamento 1, 90134 Palermo, Italy \\ e-mail: stelzer@oapa.inaf.it \\ 3 Space Science Institute, 4750 Walnut Street, Suite 205, Boulder, CO 80301, USA \\ e-mail: travis@spacescience.org \\ ${ }^{4}$ Stellar Astrophysics Centre (SAC), Department of Physics and Astronomy, Aarhus University, Ny Munkegade 120, \\ 8000 Aarhus C, Denmark
}

Received 1 March 2013 / Accepted 8 April 2013

\section{ABSTRACT}

\begin{abstract}
Context. The shortest chromospheric (Ca II H\&K) activity cycle (1.6 yr) has been recently discovered in the young ( 600 Myr) solarlike star $\iota$ Hor. Coronal X-ray activity cycles have only been discovered in a few stars other than the Sun, all of them with an older age and a lower activity level than $\iota$ Hor.

Aims. We intended to find the X-ray coronal counterpart of the chromospheric cycle for $\iota$ Hor. This represents the first X-ray cycle observed in an active star, as well as the paradigm of the first coronal cycles in the life of a solar-like star.

Methods. We monitored $\iota$ Hor with XMM-Newton observations spanning almost two years. The spectra of each observation are fit with two-temperature coronal models to study the long-term variability of the star.

Results. We find a cyclic behavior in X-rays very similar to the contemporaneous chromospheric cycle. The continuous chromospheric monitoring for more than three cycle lengths shows a trend toward decreasing amplitude, apparently modulated by a longer term trend. The second cycle is disrupted prior to reaching its maximum, followed by a brief episode of chaotic variability before the cyclic behavior resumes, only to be disrupted again after slightly more than one cycle.

Conclusions. We confirm the presence of an activity cycle of $\sim 1.6 \mathrm{yr}$ in $\iota$ Hor both in X-rays and Ca II H\&K. It is likely subject to the modulation of a longer, not yet constrained second cycle. The $1.6 \mathrm{yr}$ cycle is the shortest coronal one observed to date, and $\iota$ Hor represents the most active star for which a coronal activity cycle has been found. This cycle is probably representative of the first coronal cycles in the life of a solar-like star, at the age when life started on Earth.
\end{abstract}

Key words. stars: activity - stars: coronae - stars: chromospheres - planetary systems - stars: individual: $\iota$ Horologi - astrobiology

\section{Introduction}

Activity cycles are commonly found among late-type stars through chromospheric indicators, such as the Mount Wilson Ca II H\&K S-index. Baliunas et al. (1995) find that $\sim 60 \%$ of the main-sequence stars with spectral types from $\mathrm{F}$ to early $\mathrm{M}$ show cycles with periods in the range 2.5-25 yr. Cycles are most often found among moderately active stars. Very active stars tend to show irregular chromospheric variability rather than cycles, while inactive stars are found in "Maunder-minimum like" stages. The Sun is well known to display a Ca II H\&K cycle of $11 \mathrm{yr}$ (e.g. White et al. 1992), which is also observed in the corona through X-ray emission, spanning more than one dex (Orlando et al. 2001; Judge et al. 2003).

In recent years X-ray counterparts of the Ca II H\&K activity cycles have been detected in three binary stars: HD 81809 (Favata et al. 2004, 2008), 61 Cyg A (Hempelmann et al. 2006; Robrade et al. 2012), and $\alpha$ Cen B (Robrade et al. 2005, 2012; Ayres 2009; DeWarf et al. 2010). The two components of $61 \mathrm{Cyg}$ and $\alpha$ Cen have separations that are large enough to be resolved in X-rays but an activity cycle has so far only been established for one of the two stars in each binary. HD 81809 is

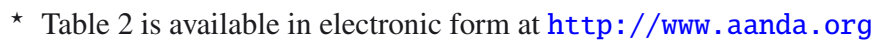

a subarcsecond binary and is unresolved in both X-rays and Ca II H\&K. All these stars have long rotation periods, estimated ages of a few Gyrs (Barnes 2007), and intermediate- to lowactivity levels. Seasonal changes have been observed in the $\mathrm{X}$-ray emission level of some younger and more active stars, such as EK Dra (Güdel 2004) and AB Dor (Sanz-Forcada et al. 2007), but no cyclic variability has been established for them. The extension of the solar-stellar analogy towards young solar analogs and towards more active stars is very important for our understanding of the early evolution of planets and life on them (e.g. Guinan et al. 2003). The study of solar analogs in different evolutionary stages indicates that the Sun at a younger age was a faster rotator with a higher activity level. Connections between solar activity and Earth's climate have been discussed in the literature (Friis-Christensen \& Lassen 1991). A stronger impact is expected for the past, given the higher UV flux arriving in the Earth's atmosphere as a result of the stronger activity (Cnossen et al. 2007). We present here the first detection of an X-ray activity cycle in a young planet-hosting star, $\iota$ Hor.

The young solar-like star $\iota$ Hor (HD 17051, HR 810), has the mass and $T_{\text {eff }}$ of an F8V star (Vauclair et al. 2008), at a HIPPARCOS distance of $17.24 \pm 0.16 \mathrm{pc}$ (van Leeuwen 2007). It has been proposed as a member of the Hyades cluster (Montes et al. 2001; Metcalfe et al. 2010), which would imply an age 
Table 1. XMM-Newton observation log, average exposure times of the three EPIC instruments, and results of spectral fits.

\begin{tabular}{lcccc}
\hline \hline Date & $\begin{array}{c}t_{\exp } \\
(\mathrm{ks})\end{array}$ & $\begin{array}{c}\log T \\
(\mathrm{~K})\end{array}$ & $\begin{array}{c}\log E M \\
\left(\mathrm{~cm}^{-3}\right)\end{array}$ & $L_{\mathrm{X}}{ }^{a}$ \\
\hline $2011-05-16$ & 6.5 & $6.47_{-0.04}^{+0.06}, 6.82_{-0.02}^{+0.03}$ & $51.12_{-0.05}^{+0.08}, 50.89_{-0.17}^{+0.07}$ & 6.0 \\
$2011-06-11$ & 11.5 & $6.54_{-0.01}^{+0.01}, 6.93_{-0.02}^{+0.02}$ & $51.32_{-0.02}^{+0.02}, 50.74_{-0.07}^{+0.06}$ & 7.4 \\
$2011-07-09$ & 8.4 & $6.50_{-0.03}^{+0.03}, 6.84_{-0.02}^{+0.03}$ & $51.18_{-0.04}^{+0.05}, 50.84_{-0.11}^{+0.07}$ & 6.3 \\
$2011-08-04$ & 7.7 & $6.56_{-0.01}^{+0.01}, 6.96_{-0.02}^{+0.03}$ & $51.33_{-0.03}^{+0.03}, 50.78_{-0.07}^{+0.06}$ & 7.8 \\
$2011-11-20$ & 6.9 & $6.56_{-0.02}^{+0.02}, 6.97_{-0.02}^{+0.03}$ & $51.27_{-0.03}^{+0.03}, 50.85_{-0.06}^{+0.06}$ & 7.5 \\
$2011-12-18$ & 6.3 & $6.54_{-0.02}^{+0.02}, 6.96_{-0.03}^{+0.04}$ & $51.23_{-0.03}^{+0.03}, 50.60_{-0.11}^{+0.08}$ & 5.8 \\
$2012-01-15$ & 6.0 & $6.46_{-0.07}^{+0.05}, 6.82_{-0.02}^{+0.02}$ & $51.00_{-0.07}^{+0.06}, 50.86_{-0.10}^{+0.07}$ & 5.1 \\
$2012-02-10$ & 9.0 & $6.47_{-0.04}^{+0.03}, 6.80_{-0.03}^{+0.02}$ & $51.06_{-0.05}^{+0.04}, 50.62_{-0.11}^{+0.10}$ & 4.2 \\
$2012-05-19$ & 7.7 & $6.43_{-0.04}^{+0.04}, 6.83_{-0.02}^{+0.02}$ & $51.06_{-0.05}^{+0.05}, 50.73_{-0.10}^{+0.07}$ & 4.6 \\
$2012-06-29$ & 11.4 & $6.44_{-0.05}^{+0.03}, 6.83_{-0.01}^{+0.02}$ & $51.09_{-0.04}^{+0.04}, 50.85_{-0.06}^{+0.06}$ & 5.5 \\
$2012-08-09$ & 8.1 & $6.44_{-0.05}^{+0.04}, 6.82_{-0.01}^{+0.02}$ & $51.05_{-0.05}^{+0.05}, 50.83_{-0.08}^{+0.06}$ & 5.1 \\
$2012-11-18$ & 5.3 & $6.48_{-0.05}^{+0.07}, 6.83_{-0.02}^{+0.09}$ & $51.15_{-0.06}^{+0.10}, 50.78_{-0.34}^{+0.09}$ & 5.6 \\
$2012-12-20$ & 5.5 & $6.46_{-0.07}^{+0.05}, 6.83_{-0.02}^{+0.02}$ & $51.04_{-0.07}^{+0.06}, 50.89_{-0.09}^{+0.07}$ & 5.5 \\
$2013-02-03$ & 7.6 & $6.46_{-0.05}^{+0.04}, 6.84_{-0.02}^{+0.02}$ & $51.05_{-0.06}^{+0.05}, 50.81_{-0.08}^{+0.07}$ & 5.0 \\
\hline
\end{tabular}

Notes. ${ }^{(a)} L_{\mathrm{X}}\left(\times 10^{28} \mathrm{erg} \mathrm{s}^{-1}\right)$ has statistical errors in the range $0.05-0.08$.

of $\sim 625 \mathrm{Myr}$ (Lebreton et al. 2001), so roughly consistent with the age of $\sim 500 \mathrm{Myr}$ calculated from the X-ray emission by Sanz-Forcada et al. (2011), $625 \mathrm{Myr}$ from asteroseismology (Vauclair et al. 2008), and 740 Myr from gyrochronology (Barnes 2007). It hosts a planet with $M \sin i=2.26 M_{\mathrm{J}}$ orbiting at a separation of $0.92 \mathrm{AU}$ (Kürster et al. 2000). A chromospheric cycle with a period of only $1.6 \mathrm{yr}$ has been identified by Metcalfe et al. (2010) in Ca II H\&K measurements from lowresolution spectra collected between 2008 and 2010. This is the shortest activity cycle known to date.

Following the discovery of the chromospheric cycle of $\iota$ Hor, we started monitoring this star with XMM-Newton in 2011. The Ca II H\&K observations have continued throughout this period. We present here the analysis of the long-term variability from the $\mathrm{X}$-ray and $\mathrm{Ca}$ II H\&K data collected over the past two years. A deeper analysis of the X-ray data will follow in a future publication. In Sect. 2 we describe the observations. The results are given in Sect. 3, and Sect. 4 presents the discussion and conclusions.

\section{Observations}

\subsection{Coronal $X$-rays}

Between May 2011 and February 2013 we obtained 14 snapshots of $\iota$ Hor (XMM prop. ID \#067361, \#069355, P. I. Sanz-Forcada, and a DDT observation on Feb. 3 2013). The observing $\log$ is presented in Table 1. Data were reduced following standard procedures in the software SAS v12.0.1. After removing time intervals affected by high background, the individual exposure times were between $5 \mathrm{ks}$ and $11 \mathrm{ks}$. Light curves of the three XMM-Newton/EPIC detectors (spectral range 0.1-15 keV, $E / \Delta E \sim 20-50$, Turner et al. 2001; Strüder et al. 2001) were extracted separately for each of the three EPIC instruments to identify eventual short-term variations such as flares. The ISIS package (Houck \& Denicola 2000) and the Astrophysics Plasma Emission Database (APED v2.0.2, Foster et al. 2012) were used to simultaneously fit the EPIC (pn, MOS1 and MOS2) spectra of each epoch with two-temperature models. Next to the two temperatures, in the initial fits we let the overall stellar

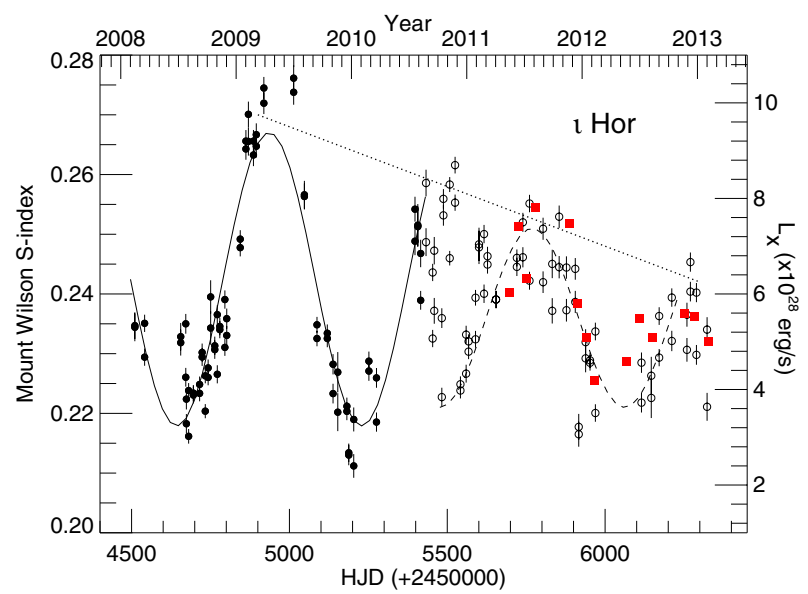

Fig. 1. Time series for the CaIIH\&K $S$-index of $\iota$ Hor. The solid line represents the cycle calculated by Metcalfe et al. (2010) for the data shown with filled circles. The new data are shown with open plotting symbols. A dashed line follows the new cycle starting after HJD 2455 500, with the same periodicity. Red filled squares represent the X-ray luminosity of the coronal observations. A dotted line is displayed marking the long-term trend of the cycle maxima.

metallicity and the oxygen abundance be free parameters, resulting in fits with unconstrained abundances in a few time intervals. We then fixed these two parameters to their median values $([\mathrm{M} / \mathrm{H}]=0.1,[\mathrm{O} / \mathrm{H}]=-0.44)$ of all observations, and performed a new spectral fit for each observation. The best-fit temperatures $\left(T_{1}, T_{2}\right)$ and emission measures $\left(E M_{1}, E M_{2}\right)$ are given in Table 1 . The spectral analysis also yields the X-ray flux for the individual observations from which we computed the X-ray luminosities listed in Table 1, calculated in the canonical ROSAT band $0.12-2.48 \mathrm{keV}(5-100 \AA)$, with error bars based on the signal-to-noise ratio. The surface flux $\left(F_{\mathrm{X}}\right)$ was calculated using a stellar radius of $R=1.18 R_{\odot}$ (Metcalfe et al. 2010).

\subsection{Chromospheric Ca II H\&K}

Observations of the Ca II H\&K lines for $\iota$ Hor were collected as part of the SMARTS Southern H\&K project (Metcalfe et al. 2009), a time-domain survey of stellar activity variations for the brightest stars in the southern hemisphere. The discovery observations for the 1.6-year activity cycle in $\iota$ Hor included $74 \mathrm{spec}$ tra spanning 37 epochs between Feb. 15, 2008 and Aug. 09, 2010. Monitoring continued after the discovery with 70 additional spectra collected on 35 epochs from Aug. 26, 2010 through Feb. 1, 2013, shortly before the RC Spec instrument was decommissioned and the SMARTS Southern H\&K project officially ended. Details of the data reduction and calibration procedure can be found in Metcalfe et al. (2010), and the new observations are tabulated in the online material (see Table 2).

\section{Results}

Figure 1 shows the full CaIIH\&K time series of $\iota$ Hor from 2008, the beginning of the optical spectroscopic monitoring, until the time of writing. The data collected between 2008 and the middle of 2010 are represented in Fig. 1, with the $1.6 \mathrm{yr}$ cycle derived for these data by Metcalfe et al. (2010) overplotted. After this time span, the clearly periodic behavior of the $S$-index was replaced by erratic Ca II H\&K variations. A few months later, the cyclic variability seems to have returned with a lower amplitude. We plot in Fig. 1 the new apparent cycle, with the same period as before but shifted in phase and with smaller 


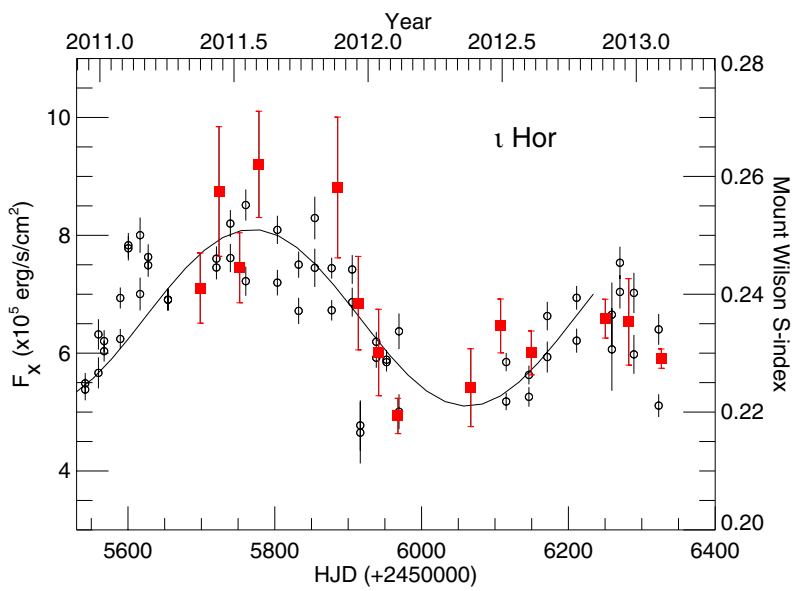

Fig. 2. Time series of coronal surface flux (filled squares) and chromospheric S-index (open dots) for $\iota$ Hor. The solid line indicates the cycle calculated in Metcalfe et al. (2010), shifted by 395 d. Error bars of coronal X-ray surface flux are based on flux variations within each snapshot.

amplitude. The new cycle seems to stop near the end of 2012, when the activity decreases again instead of the expected increase. The successive maxima, connected in Fig. 1, describe a modulation by a longer term trend, possibly a second cycle.

The XMM-Newton data cover the time range of the second calcium cycle from mid-2011 until the present (February 2013). The mean X-ray luminosities from all snapshots are displayed together with the Ca II H\&K $S$-index in Fig. 1, and the surface $\mathrm{X}$-ray flux is shown in Fig. 2. The emission measure and average temperature reflect the same pattern. The X-ray coronal emission mimics the Ca II chromospheric cycle, and it decreases at the end of the interval, following the chromospheric behavior. The error bars for the X-ray measurements in Fig. 2 represent the variations observed within each 5-11 ks snapshot ${ }^{1}$. No strong flares are seen in these light curves, but small-scale X-ray variability is present (Sanz-Forcada et al., in prep.). This short-term variability decreases at times of lower X-ray luminosity, i.e. near the cycle minimum. The cyclic behavior is conditioned by the longer term trend, which hampers the calculation of the X-ray cycle length. The coronal cycle does not show any essential differences with respect to its chromospheric counterpart.

The characteristic parameters of the chromospheric (Ca II H\&K) and the coronal (X-ray) cycle of $\iota$ Hor observed since HJD 2455550 (end of 2010) are indicated below. The coronal activity index has an average value of $\log L_{\mathrm{X}} / L_{\mathrm{bol}}=$ $-5.0\left(\log L_{\mathrm{bol}}[\mathrm{erg} / \mathrm{s}]=33.8\right.$, Sanz-Forcada et al. 2011), spanning 0.27 dex in amplitude. In the chromosphere we observe $\log R_{\mathrm{H} \& \mathrm{~K}}^{\prime}=-4.6$ as an average, with 0.2 dex spanned since the end of 2010. The parameter $R_{\mathrm{H} \& \mathrm{~K}}^{\prime}$ is obtained from the $S$-index using the calibration presented by Noyes et al. (1984) and includes subtraction of the photospheric contribution.

\section{Discussion and conclusions}

Iota Hor is the as yet youngest and most active star on which both a chromospheric and a coronal activity cycle have been detected so far. Given the F8 V spectral type and $~ 600 \mathrm{Myr}$ age of $\iota$ Hor, these observations might represent a paradigm of the first activity cycles in the life of a solar-like star.

\footnotetext{
1 Light curves of each observation were generated using $1000 \mathrm{~s}$ bins (Sanz-Forcada et al., in prep.). The maximum and minimum of the values within each observation are used to establish the "error bars" around a central point.
}

We find that both diagnostics, X-rays and Ca II H\&K, reveal evidence for changes in the activity cycle of $\iota$ Hor on a timescale comparable to the cycle duration. The variability observed in Fig. 1, and described in Sect. 3, draws a scenario with a short (1.6 yr) cycle that shows some irregularities. A possible explanation is a second, longer cycle superposed onto the $1.6 \mathrm{yr}$ variation and modulating the shorter cycle. Extending the time baseline of monitoring observations to a few more years will shed light on this interpretation. The presence of more than one activity cycle persisting on the same star at a given time has been noted in Ca II H\&K data for other active stars (e.g., Baliunas \& Soon 1995), while changes in the periodicity of the cycles have been observed in $\mathrm{Ca}$ II H\&K and photometric data of a few stars, including the Sun (Oláh et al. 2009).

Altogether, $\iota$ Hor has now been monitored for approximately three cycle lengths, and there have been two episodes where the periodic behavior was disrupted. Since some time after the disruption, the cycles resumes with the same period, it seems that the dynamo process itself is disturbed by some unknown mechanism. Interaction with a binary companion would be a possible cause, but $\iota$ Hor can be considered to be a single star given its extensive spectroscopic monitoring that has led to the discovery of its planet. We have noticed that the anomaly in the $\iota$ Hor activity cycle in 2010 occurs at the orbital phase ${ }^{2} \phi \sim 0$ of the planet $\iota$ Hor b. This coincidence happens again shortly after the disruption of the cycle in 2012. Although some influence of the positions of the solar system giant planets in the activity cycle of the Sun has been proposed recently (Abreu et al. 2012), it seems unlikely to us that the planet (at $0.9 \mathrm{AU}$ ) has any influence on the stellar cycle of $\iota$ Hor. The orbital parameters of Naef et al. (2001) do not show any coincidence. An alternative explanation would relate the observed behavior with the geometry of the star. In the Sun, the two hemispheres can drive cycles that are out of phase with each other (e.g. Ribes \& Nesme-Ribes 1993), which can lead to phenomena like the recent extended minimum. In $\iota$ Hor, with an inclination $i \sim 60^{\circ}$, we are primarily seeing one magnetic pole at a time, so it appears that one of the magnetic poles may be driving a coherent cycle, while the other is doing something more chaotic. Because we can observe some fraction of the less visible hemisphere, we see more than $50 \%$ of the true cycle period as the coherent pole drives a cycle first in the more visible hemisphere and then in the less visible hemisphere. Eventually the activity belt migrates out of view in the less visible hemisphere, and we are left with only the chaotic hemisphere.

According to the relations between X-ray luminosity and rotation given by Pizzolato et al. (2003) our mean value $\left(L_{X}=\right.$ $\left.6 \times 10^{28} \mathrm{erg} / \mathrm{s}\right)$ corresponds to a rotation period of $\sim 9 \mathrm{~d}$. This is in fair agreement with the period derived from Ca II H\&K measurements of $\sim 8 \mathrm{~d}$ (Metcalfe et al. 2010; Boisse et al. 2011). The ratio between rotation and activity cycle period has gained attention in the literature because it constrains the strength of the $\alpha$-effect in kinematic dynamo theory. A positive correlation between observed values for $P_{\text {rot }}$ and $P_{\text {cyc }}$ derived for a subsample from the Mt.Wilson project with reliable cycles has been established by various authors (e.g., Brandenburg et al. 1998; Saar \& Brandenburg 1999; Böhm-Vitense 2007). These studies identified different branches for active and inactive stars that are separated by $\log R_{\mathrm{H} \& \mathrm{~K}}^{\prime}=-4.75$. A large fraction of the active group, represented by faster rotators, displays two activity cycles. For these stars the shorter cycle lies on the extension of the inactive branch to short rotation periods. This relation has been

$2 \phi=0$ is defined by Kürster et al. (2000) as the time of maximum radial velocity of the planet as seen from Earth. 


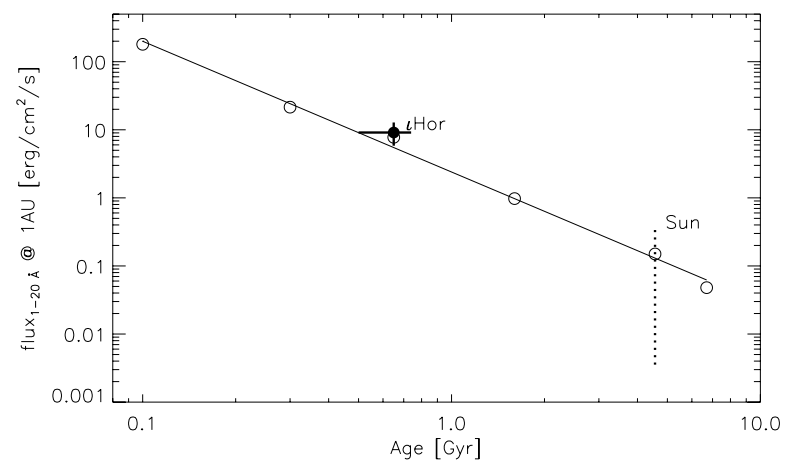

Fig. 3. X-ray flux in 1-20 $\AA$ versus stellar age for solar analogs. The six stars studied by Ribas et al. (2005), including the Sun, are shown as open circles and the corresponding power law fit as a solid line. $\iota$ Hor is displayed with a filled circle. Vertical lines in $\iota$ Hor and the Sun account for their activity cycle as measured in the ASCA $0.5-10 \mathrm{keV}$ band (Orlando et al. 2001). All fluxes have been normalized to a distance of $1 \mathrm{AU}$ from the star and a stellar radius of $1 R_{\odot}$.

reproduced by Lorente \& Montesinos (2005) with a local solution for an interface dynamo. $\iota$ Hor fits into this picture well. Its mean chromospheric activity $\left(\log \left\langle R_{\mathrm{H} \& \mathrm{~K}}^{\prime}\right\rangle=-4.6\right)$ puts it in the active group. The measured values for its rotation $\left(P_{\text {rot }} \sim 8 \mathrm{~d}\right)$ and cycle $\left(P_{\text {cyc }}=1.6 \mathrm{yr}\right)$ periods are in excellent agreement with the linear regression derived by Lorente \& Montesinos (2005) for which $\iota$ Hor to date defines the low-period end. Moreover, we found indications of a longer, as yet unconstrained cycle in $\iota$ Hor in the form of a decreasing amplitude of its $1.6 \mathrm{yr}$ signal.

Coronal activity cycles in planet-host stars such as $\iota$ Hor, are of particular interest given the disputed evidence of a connection between solar cycle variations and Earth's climate (Friis-Christensen \& Lassen 1991). From an astrobiological point of view, the case of $\iota$ Hor is interesting because it represents a young solar-like star at an age of $\sim 600 \mathrm{Myr}$, coincident with the time at which life started to develop on Earth. The high-energy radiation from the Sun may have played an important role during initial steps in the evolution of life (e.g. Cnossen et al. 2007, and references therein). Winds and highenergy radiation of the young Sun have also been held responsible for processes on other solar system planets, e.g. the erosion of Mercury's early atmosphere (Guinan \& Ribas 2004). Similarly the amount of UV and XUV radiation and its variation in time can be considered crucial for the evolution of the atmospheres and for the development of life on extrasolar planets (Sanz-Forcada et al. 2011). Ribas et al. (2005) have compared the XUV fluxes of six solar analogs, including the Sun itself, spanning an age range from 100 Myr to 6.7 Gyr. They observed a power-law decrease of the high-energy emission for all wavelength ranges between $1 \AA$ and $\sim 1200 \AA$. Figure 3 shows $\iota$ Hor, together with the data from Ribas et al. (2005), for the X-ray band (1-20 ̊ range). The position of $\iota$ Hor agrees well with this relation.

Activity cycles add a shorter term modulation to the amount of high-energy radiation arriving in the planet atmosphere. An active star like $\iota$ Hor is expected to have a large area of its surface covered with active regions even at the minimum of its cycle. This might explain why a coronal cycle only results in an increase in the X-ray emission by a factor of two. It is presently unclear if the amplitude of these modulations depends on stellar age, since only two solar-like stars with known age have measured X-ray activity cycles. For $\iota$ Hor, this variation may be minor compared to large flares and star-to-star variations. In contrast, almost two orders of magnitude are spanned by the solar coronal cycle (see Fig. 3). Finally, the $\iota$ Hor system may in the future allow us to track the effects of the stellar radiation on a giant planet at the Sun-Earth distance.

Acknowledgements. We acknowledge Norbert Schartel for the last observation, granted as XMM-Newton Director Discretionary Time (DDT). J.S.F. acknowledge support from the Spanish MICINN through grant AYA2008-02038 and AYA2011-30147-C03-03. The Southern H\&K project was supported under the NOAO long-term program 2011B-0001 with additional time from SMARTS partner institutions. B.S. acknowledges support by the Faculty of the European Space Astronomy Center (ESAC). We also thank B. Montesinos and Billy T. Orlando for useful conversations on the topic.

\section{References}

Abreu, J. A., Beer, J., Ferriz-Mas, A., McCracken, K. G., \& Steinhilber, F. 2012, A\&A, 548, A88

Ayres, T. R. 2009, ApJ, 696, 1931

Baliunas, S., \& Soon, W. 1995, ApJ, 450, 896

Baliunas, S. L., Donahue, R. A., Soon, W. H., et al. 1995, ApJ, 438, 269

Barnes, S. A. 2007, ApJ, 669, 1167

Böhm-Vitense, E. 2007, ApJ, 657, 486

Boisse, I., Bouchy, F., Hébrard, G., et al. 2011, A\&A, 528, A4

Brandenburg, A., Saar, S. H., \& Turpin, C. R. 1998, ApJ, 498, L51

Cnossen, I., Sanz-Forcada, J., Favata, F., et al. 2007, J. Geophys. Res. (Planets), 112, E02008

DeWarf, L. E., Datin, K. M., \& Guinan, E. F. 2010, ApJ, 722, 343

Favata, F., Micela, G., Baliunas, S. L., et al. 2004, A\&A, 418, L13

Favata, F., Micela, G., Orlando, S., et al. 2008, A\&A, 490, 1121

Foster, A. R., Ji, L., Smith, R. K., \& Brickhouse, N. S. 2012, ApJ, 756, 128

Friis-Christensen, E., \& Lassen, K. 1991, Science, 254, 698

Güdel, M. 2004, A\&ARv, 12, 71

Guinan, E. F., \& Ribas, I. 2004, in Stars as Suns: Activity, Evolution and Planets, eds. A. K. Dupree, \& A. O. Benz, IAU Symp., 219, 423

Guinan, E. F., Ribas, I., \& Harper, G. M. 2003, ApJ, 594, 561

Hempelmann, A., Robrade, J., Schmitt, J. H. M. M., et al. 2006, A\&A, 460, 261

Houck, J. C., \& Denicola, L. A. 2000, in Astronomical Data Analysis Software and Systems IX, eds. N. Manset, C. Veillet, \& D. Crabtree, ASP Conf. Ser., 216,591

Judge, P. G., Solomon, S. C., \& Ayres, T. R. 2003, ApJ, 593, 534

Kürster, M., Endl, M., Els, S., et al. 2000, A\&A, 353, L33

Lebreton, Y., Fernandes, J., \& Lejeune, T. 2001, A\&A, 374, 540

Lorente, R., \& Montesinos, B. 2005, ApJ, 632, 1104

Metcalfe, T. S., Judge, P. G., Basu, S., et al. 2009, in Solar Analogs II workshop [arXiv: 0909.5464]

Metcalfe, T. S., Basu, S., Henry, T. J., et al. 2010, ApJ, 723, L213

Montes, D., López-Santiago, J., Gálvez, M. C., et al. 2001, MNRAS, 328, 45

Naef, D., Mayor, M., Pepe, F., et al. 2001, A\&A, 375, 205

Noyes, R. W., Hartmann, L. W., Baliunas, S. L., Duncan, D. K., \& Vaughan, A. H. 1984, ApJ, 279, 763

Oláh, K., Kolláth, Z., Granzer, T., et al. 2009, A\&A, 501, 703

Orlando, S., Peres, G., \& Reale, F. 2001, ApJ, 560, 499

Pizzolato, N., Maggio, A., Micela, G., Sciortino, S., \& Ventura, P. 2003, A\&A, 397,147

Ribas, I., Guinan, E. F., Güdel, M., \& Audard, M. 2005, ApJ, 622, 680

Ribes, J. C., \& Nesme-Ribes, E. 1993, A\&A, 276, 549

Robrade, J., Schmitt, J. H. M. M., \& Favata, F. 2005, A\&A, 442, 315

Robrade, J., Schmitt, J. H. M. M., \& Favata, F. 2012, A\&A, 543, A84

Saar, S. H., \& Brandenburg, A. 1999, ApJ, 524, 295

Sanz-Forcada, J., Micela, G., \& Maggio, A. 2007, in XMM-Newton: The Next Decade, 3

Sanz-Forcada, J., Micela, G., Ribas, I., et al. 2011, A\&A, 532, A6

Strüder, L., Briel, U., Dennerl, K., et al. 2001, A\&A, 365, L18

Turner, M. J. L., Abbey, A., Arnaud, M., et al. 2001, A\&A, 365, L27

van Leeuwen, F. 2007, A\&A, 474, 653

Vauclair, S., Laymand, M., Bouchy, F., et al. 2008, A\&A, 482, L5

White, O. R., Skumanich, A., Lean, J., Livingston, W. C., \& Keil, S. L. 1992, PASP, 104, 1139 
J. Sanz-Forcada et al.: ८ Hor, the first coronal activity cycle in a young solar-like star

Table 2. Ca II H\&K observation log, and measured Mount-Wilson S-index.

\begin{tabular}{|c|c|c|c|c|}
\hline Date & $\begin{array}{l}\text { Time (UT) } \\
\text { HH MM SS }\end{array}$ & $\begin{array}{c}\mathrm{BJD}^{a} \\
(2450000+)\end{array}$ & S-index & $\sigma_{\mathrm{S}}$ \\
\hline 2010 Aug. 26 & 062410 & 5434.76985 & 0.2487 & 0.0028 \\
\hline 2010 Aug. 26 & 062524 & 5434.77070 & 0.2586 & 0.0033 \\
\hline 2010 Sep. 16 & 060813 & 5455.75918 & 0.2436 & 0.0044 \\
\hline 2010 Sep. 16 & 060927 & 5455.76004 & 0.2325 & 0.0040 \\
\hline 2010 Sep. 21 & 073301 & 5460.81813 & 0.2372 & 0.0025 \\
\hline 2010 Sep. 21 & 073415 & 5460.81898 & 0.2472 & 0.0028 \\
\hline 2010 Oct. 15 & 070042 & 5484.79563 & 0.2227 & 0.0032 \\
\hline 2010 Oct. 15 & 070156 & 5484.79648 & 0.2360 & 0.0041 \\
\hline 2010 Oct. 20 & 024200 & 5489.61591 & 0.2559 & 0.0019 \\
\hline 2010 Oct. 20 & 024314 & 5489.61676 & 0.2532 & 0.0019 \\
\hline 2010 Nov. 09 & 035355 & 5509.66538 & 0.2460 & 0.0017 \\
\hline 2010 Nov. 09 & 035509 & 5509.66623 & 0.2583 & 0.0019 \\
\hline 2010 Nov. 26 & 040510 & 5526.67258 & 0.2553 & 0.0021 \\
\hline 2010 Nov. 26 & 040624 & 5526.67344 & 0.2616 & 0.0022 \\
\hline 2010 Dec. 13 & 051235 & 5543.71865 & 0.2249 & 0.0018 \\
\hline 2010 Dec. 13 & 051349 & 5543.71951 & 0.2238 & 0.0018 \\
\hline 2010 Dec. 31 & 024740 & 5561.61715 & 0.2332 & 0.0026 \\
\hline 2010 Dec. 31 & 024854 & 5561.61800 & 0.2267 & 0.0026 \\
\hline 2011 Jan. 08 & 030230 & 5569.62706 & 0.2321 & 0.0019 \\
\hline 2011 Jan. 08 & 030344 & 5569.62792 & 0.2303 & 0.0018 \\
\hline 2011 Jan. 30 & 033414 & 5591.64809 & 0.2394 & 0.0018 \\
\hline 2011 Jan. 30 & 033528 & 5591.64894 & 0.2324 & 0.0017 \\
\hline 2011 Feb. 10 & 030739 & 5602.62921 & 0.2483 & 0.0021 \\
\hline 2011 Feb. 10 & 030854 & 5602.63007 & 0.2478 & 0.0021 \\
\hline 2011 Feb. 26 & 005742 & 5618.53847 & 0.2500 & 0.0030 \\
\hline 2011 Feb. 26 & 005856 & 5618.53932 & 0.2400 & 0.0028 \\
\hline 2011 Mar. 09 & 004939 & 5629.53263 & 0.2449 & 0.0020 \\
\hline 2011 Mar. 09 & 005053 & 5629.53348 & 0.2463 & 0.0022 \\
\hline 2011 Apr. 04 & 232118 & 5656.47110 & 0.2391 & 0.0019 \\
\hline 2011 Apr. 04 & 232232 & 5656.47196 & 0.2391 & 0.0018 \\
\hline 2011 Jun. 10 & 102505 & 5722.93392 & 0.2445 & 0.0020 \\
\hline 2011 Jun. 10 & 102620 & 5722.93478 & 0.2460 & 0.0021 \\
\hline 2011 Jun. 29 & 095907 & 5741.91677 & 0.2461 & 0.0024 \\
\hline 2011 Jun. 29 & 100021 & 5741.91762 & 0.2520 & 0.0023 \\
\hline 2011 Jul. 20 & 090957 & 5762.88359 & 0.2422 & 0.0025 \\
\hline 2011 Jul. 20 & 091111 & 5762.88445 & 0.2551 & 0.0026 \\
\hline 2011 Sep. 01 & 075947 & 5805.83640 & 0.2509 & 0.0024 \\
\hline 2011 Sep. 01 & 080102 & 5805.83726 & 0.2420 & 0.0022 \\
\hline 2011 Sep. 30 & 093844 & 5834.90546 & 0.2372 & 0.0022 \\
\hline 2011 Sep. 30 & 093958 & 5834.90632 & 0.2450 & 0.0022 \\
\hline 2011 Oct. 22 & 072115 & 5856.80980 & 0.2529 & 0.0036 \\
\hline 2011 Oct. 22 & 072229 & 5856.81066 & 0.2445 & 0.0033 \\
\hline 2011 Nov. 14 & 055140 & 5879.74699 & 0.2444 & 0.0018 \\
\hline 2011 Nov. 14 & 055254 & 5879.74785 & 0.2373 & 0.0017 \\
\hline 2011 Dec. 12 & 022247 & 5907.60081 & 0.2387 & 0.0024 \\
\hline 2011 Dec. 12 & 022402 & 5907.60166 & 0.2442 & 0.0024 \\
\hline 2011 Dec. 23 & 021540 & 5918.59534 & 0.2165 & 0.0052 \\
\hline 2011 Dec. 23 & 021654 & 5918.59619 & 0.2177 & 0.0043 \\
\hline 2012 Jan. 14 & 041910 & 5940.68003 & 0.2319 & 0.0017 \\
\hline 2012 Jan. 14 & 042024 & 5940.68089 & 0.2292 & 0.0017 \\
\hline 2012 Jan. 28 & 022714 & 5954.60166 & 0.2285 & 0.0016 \\
\hline 2012 Jan. 28 & 022828 & 5954.60252 & 0.2289 & 0.0016 \\
\hline 2012 Feb. 14 & 013913 & 5971.56766 & 0.2337 & 0.0030 \\
\hline 2012 Feb. 14 & 014027 & 5971.56851 & 0.2201 & 0.0030 \\
\hline 2012 Jul. 09 & 083820 & 6117.86119 & 0.2218 & 0.0015 \\
\hline 2012 Jul. 09 & 083935 & 6117.86204 & 0.2285 & 0.0015 \\
\hline 2012 Aug. 09 & 091310 & 6148.88669 & 0.2226 & 0.0017 \\
\hline 2012 Aug. 09 & 091424 & 6148.88755 & 0.2263 & 0.0016 \\
\hline 2012 Sep. 03 & 085351 & 6173.87402 & 0.2363 & 0.0024 \\
\hline 2012 Sep. 03 & 085505 & 6173.87488 & 0.2293 & 0.0026 \\
\hline 2012 Oct. 13 & 070734 & 6213.80043 & 0.2321 & 0.0020 \\
\hline 2012 Oct. 13 & 070848 & 6213.80129 & 0.2394 & 0.0020 \\
\hline 2012 Nov. 30 & 052913 & 6261.73078 & 0.2365 & 0.0055 \\
\hline 2012 Nov. 30 & 053027 & 6261.73164 & 0.2306 & 0.0070 \\
\hline 2012 Dec. 11 & 031341 & 6272.63618 & 0.2404 & 0.0028 \\
\hline 2012 Dec. 11 & 031455 & 6272.63703 & 0.2453 & 0.0027 \\
\hline 2012 Dec. 30 & 045305 & 6291.70428 & 0.2298 & 0.0033 \\
\hline 2012 Dec. 30 & 045419 & 6291.70514 & 0.2402 & 0.0034 \\
\hline 2013 Feb. 01 & 022002 & 6324.59648 & 0.2340 & 0.0026 \\
\hline 2013 Feb. 01 & 022116 & 6324.59734 & 0.2211 & 0.0019 \\
\hline
\end{tabular}

Notes. ${ }^{(a)}$ Barycentric Julian date of observation. 